\title{
Different steps in the evolution of neo-sex chromosomes in two East African Spalacomimus species (Orthoptera: Tettigoniidae: Hetrodinae)
}

\author{
ELŻBIETA WARCHAŁOWSKA-ŚLIWA', BEATA GRZYWACZ ${ }^{1}$, ANNA MARYAŃSKA-NADACHOWSKA ${ }^{1}$, \\ ANDREAS HEMP ${ }^{2}$ and CLAUDia HEMP ${ }^{3}$

\begin{abstract}
${ }^{1}$ Institute of Systematics and Evolution of Animals, Polish Academy of Sciences, Kraków, Poland; e-mails: warchalowska@isez.pan.krakow.pl; grzywacz@isez.pan.krakow.pl; maryanska@isez.pan.krakow.pl ${ }^{2}$ Department of Plant Systematics, University of Bayreuth, Germany; e-mail: andreas.hemp@uni-bayreuth.de ${ }^{3}$ Department of Animal Ecology and Tropical Biology (Zoology III), University of Würzburg, Germany; e-mail: claudia.hemp@uni-wuerzburg.de
\end{abstract}

Key words. Orthoptera, Tettigoniidae, Spalacomimus, karyotype evolution, neo-XY

\begin{abstract}
Two orthopteran species belonging to the East African genus Spalacomimus, S. verruciferus and S. talpa (Tettigoniidae: Hetrodinae), were investigated using both molecular cytogenetic techniques, including fluorescence in situ hybridization with 18S rDNA and (TTAGG) telomeric probes, and classical techniques, such as C-banding, fluorochrome $\mathrm{CMA}_{3} / \mathrm{DAPI}$ staining, and silver impregnation. These techniques allowed us to identify cytogenetic markers that were then used to elucidate chromosome organization and differences between species. Chromosomal complements are reported for the first time for both analyzed species, which showed the same chromosomal number of $2 \mathrm{n}=24$ and the neo-XY sex chromosome system; however, the morphology of neo-sex chromosomes differs between these species. A cluster of the major rDNA (i.e., the nucleolar organizer region, NOR) was found to be located in the interstitial region of the sex chromosomes of $S$. verruciferus, whereas it was on the long pair of autosomes in $S$. talpa. The difference between the karyotypes of the studied species indicate divergent origins of their neo-XY systems. A detailed description of the structure and behaviour of the sex chromosome configuration in male meiosis is provided. In S. verruciferus, the neo-XY system is derived from a Robertsonian fusion between the original acrocentric $X$ and an acrocentric long pair of autosomes bearing an rDNA/NOR locus, leading to a bi-armed $\mathrm{X}$ and an acrocentric neo-Y chromosome. The neo-XY in $S$. talpa arose due to complicated rearrangements, attributable to a tandem fusion between the original acrocentric $\mathrm{X}$ and part of a medium-sized autosome, and resulting in supernumerary chromosomes. In the latter case, behaviour of the neo-X and neo-Y demonstrates a post-reductional division of these chromosomes. Variation in the evolution of two neo-XY sex chromosome systems are novel phylogenetic markers for the Hetrodinae and seem to promote speciation as important aspect for taxonomy.
\end{abstract}

\section{INTRODUCTION}

Hetrodinae are listed as a subfamily of Tettigoniidae. The group is restricted to Africa, the Arabian Peninsula, and parts of Mediterranean Europe. In this study, two species belonging to the genus Spalacomimus Karsch, a genus with the highest diversity in East Africa, were investigated cytotaxonomically. The genus Spalacomimus contains seven species, most of which are restricted to Tanzania. Generally, little is known about the distribution, habitat, and ecology of Spalacomimus species. We investigated S. talpa (Gerstaecker, 1965) known from the area of Mts Kilimanjaro and Meru (Hemp, 2013) and several other localities in East Africa. The species is nocturnal and males are found in small shrubs and trees at night, performing their calling song. S. talpa has been recorded from scattered Acacia thorn grass land, desert-grass, and thorn-bush (Chopard \& Kevan, 1954) in Kenya. In northern Tanzania, it is a common species found at submontane elevations in coffee-banana plantations on Mt Kilimanjaro, but occasionally occurs also in savanna habitats (Hemp, 2013). S. verruciferus (Karsch, 1997) is known from several localities in East Africa but beside collection data no further information is available for this species. In northern Tanzania it is a rarely collected savanna species. Similar to S. talpa, S. verruciferus males perform their calling songs hanging from low branches at night.

The chromosomes of only four African Hetrodinae species have been studied to date using classical techniques, involving only Giemsa staining (Favrelle, 1936; Matthey, 1948; Mbata, 2005). The use of a specific technique for chromosome identification (heterochromatin C-banding) has been applied only to the species Hetrodes pupus (Linnaeus) (Warchałowska-Śliwa \& Bugrov, 2009). The diploid chromosome number $(2 n)$ with acrocentric chromosomes varies from 29 in the male of Eugaster fernandezi Graells, E. guyoni Serville, and E. spinulosa (L.) (Favrelle, 1936; Matthey, 1948), to 27 in Hetrodes pupus (Warchałowska-Śliwa \& Bugrov, 2009), and to only 25 in Acanthoplus speiseri Brancsik (Mbata, 2005). All species are characterized by an X0 male/XX female sex chromosome system, which is considered ancestral for tettigoniids (e.g., Warchałowska-Śliwa, 1998).

In more than 100 orthopteran species, most of which are grasshoppers (e.g., White, 1979; Bugrov \& WarchałowskaŚliwa, 1997; Castillo et al., 2010), the neo-XY sex chromosome system has been derived from the X0/XX chromosome system. Only a few instances, this system have 
been recorded in Tettigonioidea species, so far only in Phaneropterinae (Dave, 1965; White et al., 1967a; Ferreira, 1969, 1976; Alicata et al., 1974; Messina, 1981; Warchałowska-Śliwa \& Bugrov, 1998; Webber et al., 2003; Warchałowska-Śliwa et al., 2011), some Tettigoniinae (Camacho et al., 1981), Nedubinae (Ueshima \& Rentz, 1979), Bradyporinae (Fernandez-Piqueras et al., 1981, 1982), and Listroscelidinae (White et al., 1967b). In most cases, the neo-sex chromosome $\mathrm{X}$ is bi-armed, produced by a Robertsonian (centric) fusion between an acrocentric $\mathrm{X}$ and an autosome, whereas the neo- $Y$ is usually acrocentric.

Karyotype differentiation include reductions in chromosomes number and sex chromosome rearrangements such that it has been speculated that sex chromosome-autosome fusions have played a role in speciation by causing reproductive isolation of subpopulations bearing such rearrangements (Brown \& O’Neill, 2010; Faria \& Navarro, 2010; Kitano \& Peichel, 2012; Nguyen et al., 2013). These changes are regarded by some researchers as being responsible for creating genetic organization and differences or similarities between taxa and as such may be useful in determining whether cytogenetic markers can be used for distinguishing some groups of species.

Recently, the application of different cytogenetic staining techniques (molecular and classical methods) has led to better characterization of tettigoniid karyotypes, with relevance for the identification of genus- or species-specific patterns (e.g., Warchałowska-Śliwa et al., 2009, 2011, 2013a, b; Grzywacz et al., 2014). In the present study, we present the chromosomal pattern of two Spalacomimus species. Chromosomal characters were examined using both molecular fluorescence in situ hybridization (FISH) with $18 \mathrm{~S}$ rDNA and telomeric probes (TTAGG), and conventional methods, including C-banding, fluorochrome $\mathrm{CMA}_{3} / \mathrm{DAPI}$ staining, and silver impregnation (AgNORs). This information will, we trust, provide a better understanding of the karyotype structure of both species and the relationship between them. The main objective of the present work was especially to elucidate the origin of the neo-XY sex chromosome system in the two Spalacomimus species under study.

\section{MATERIAL AND METHODS}

Cytogenetic analysis was conducted on seven adult males collected from northern Tanzania, Mt Kilimanjaro, near Lake Chala on the eastern foothills and the banana-coffee plantaion belt on the southern slopes in the area of Old Moshi. Three Spalacomimus verruciferus (Karsch, 1997) (CH7188, CH7189, February 2009; CH7637, March 2013) and four Spalacomimus talpa (Gerstaecker, 1869) (CH7354, August 2010; CH7525, December 2012; CH7626, February 2013; CH7699, September 2013) were investigated.

Chromosomal preparations were obtained from gonads and hepatic caeca. Testes and somatic body parts were excised, incubated in hypotonic solution ( $0.9 \%$ sodium citrate for $20 \mathrm{~min})$ fixed in ethanol : acetic acid ( $3: 1)$, and squashed in $45 \%$ acetic acid. Coverslips were removed using the dry ice procedure and the preparations were air dried. Constitutive heterochromatin was revealed by the C-banding technique as described by Sumner (1972) with slight modification; the slides were stained with
Giemsa after treatment with $0.2 \mathrm{~N} \mathrm{HCl}$ for $20 \mathrm{~min}$, immersed in a saturated solution of $\mathrm{Ba}(\mathrm{OH})_{2}$ at $60^{\circ} \mathrm{C}$ for $5 \mathrm{~min}$.

Karyotypes were reconstructed by arranging homologous chromosomes in order of decreasing size. Relative chromosome lengths of the diploid complement (including the sex chromosomes) were calculated as a percentage of total chromosome length (\% TCL) according to Král et al. (2006).

Some slides were stained with $\mathrm{CMA}_{3}$ to reveal GC-rich regions and with DAPI to reveal AT-rich ones (Schweizer, 1976). The silver staining method (with $\mathrm{AgNO}_{3}$ ), visualizing nucleolus organizer regions (NORs), was performed as described previously (Warchałowska-Śliwa \& Maryańska-Nadachowska, 1992). Fluorescence in situ hybridization (FISH) with ribosomal 18S DNA (rDNA) and telomeric (TTAGG) ${ }_{n}$ DNA probes was performed according to Warchałowska-Śliwa et al. (2009). The probes were prepared and labelled as described by Warchałowska-Śliwa et al. (2009), with the $18 \mathrm{~S}$ rDNA probe containing $1.8 \mathrm{~kb}$ fragments amplified from the genomic DNA of Isophya pavelii (Orthoptera, Phaneropterinae).

The chromosomes were studied using a Nikon Eclipse 400 fitted with a CCD DS-U1 camera, a set of standard filters, and an NIS-Elements BR 3.0 image-analyzing system (Nikon Eclipse 400). Images were processed and arranged with Adobe Photoshop. For each individual, at least 20 meiotic divisions (from diplotene to metaphase I) and 10 spermatogonial metaphases per male were analyzed using all the above-mentioned techniques. Additionally, at least 10 somatic metaphases were examined for the hepatic caeca of two S. talpa males (CH7626, CH7699). In both analyzed species, the rDNA-FISH pattern and the location of active NORs were always recorded for the same individual.

\section{RESULTS}

Both analyzed species showed the same chromosomal number $2 n=24$, but differed in terms of the morphology of the sex chromosomes (Figs 1-4).

\section{Autosomal complement}

The autosomes of both species could be divided into three size groups. The karyotype of $S$. verruciferus consists of one long $(9.06 \%$ of TCL), two medium $(3.67,3.57 \%$ of TCL) and eight small (from 2.06 to $0.8 \%$ of TCL) pairs (Fig. 1a,b). In turn, S. talpa was found to have one long (16.25\% of TCL), one medium (8.0\% of TCL), and nine small pairs (from $3.1 \%$ to $0.7 \%$ of TCL) (Fig. 1c, d).

In $S$. talpa, two rDNA clusters, located interstitially on the long pair, were detected (Fig. 1d). 18S rDNA coincident with active NORs was visualized by Ag-NOR staining (Fig. 1e). The signals produced by FISH with the (TTAGG) ${ }_{n}$ probe were detected at the distal ends of most autosomes. Differences in the intensity of hybridization signals were clearly seen between the homologues of the long pair in S. verruciferus (Fig. 1b).

After both C-staining and fluorochrome DAPI/CMA double-staining, chromosome regions showed some quantitative and qualitative variation between the analyzed species in terms of constitutive heterochromatin. Intraspecific heterochromatin heteromorphism was observed in some chromosomes of both species. In S. verruciferus autosomes, heterochromatin in the form of thick C-bands in the paracentromeric region was visualized with bright homogenous DAPI-positive (DAPI+, AT-rich) and $\mathrm{CMA}_{3}$-positive $\left(\mathrm{CMA}_{3}+, \mathrm{GC}\right.$-rich) signals (Fig. 2a). In the long pair, 


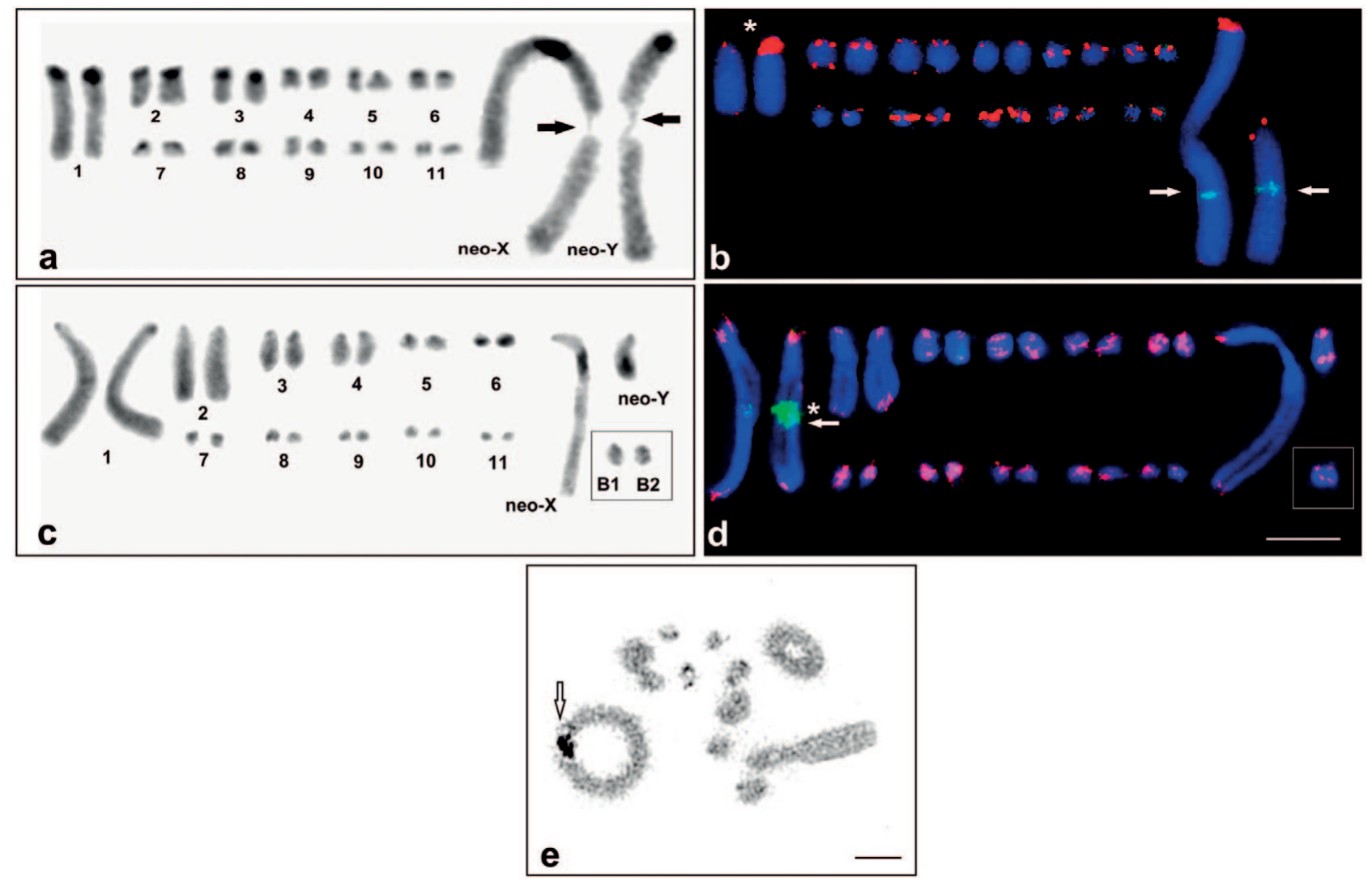

Fig. 1. Male complement subjected to C-banding (a, c), FISH with 18S rDNA (green) and telomeric DNA (red) probes (b, d), and silver staining (e) show differences between S.verruciferus (a, b) and S. talpa (c, d, e). Black arrows indicate a secondary constriction in spermatogonial metaphase (a) and white arrows rDNA sites located on both sex chromosomes (b) and on the long pair of autosomes (d). Hybridization areas varying in size between the long homologues of the two test species are marked with an asterisk (*). Open arrows indicate a pair of active NORs located in the interstitial region of the long autosome pair in metaphase I (e). The insert in the bottom right corner shows an enlarged image of the two types of B chromosomes B1 and B2 (c, d). Bar $=10 \mu \mathrm{m}$.

heterochromatin heteromorphism in respect to the pattern of C-bands, fluorochrome bands, as well as telomeric signals obtained with the (TTAGG) ${ }_{n}$ probe was observed in one out of three individuals. In contrast, in S. talpa, heterochromatin formed thin C-positive paracentromeric bands and an interstitial band on the long pair, with DAPI- and GC-rich blocks that were weakly stained by $\mathrm{CMA}_{3}$, except those present on the autosome pair 6 (thick C-positive/ DAPI+/CMA + bands). Heteromorphism of both C-bands and fluorochrome bands, similar to that of rDNA signals on the autosome pair 1, was observed in terms of the size/ strength of bands on homologues in two out of four individuals (not shown). Additionally, a secondary constriction in the same place was rarely observed (not shown).

\section{Sex chromosomes}

In $S$. verruciferus the largest elements in the karyotype were the sex chromosomes, a submetacentric neo-X (21.78\% of TCL) and an acrocentric neo-Y (13.58\% of TCL) (Fig. 1a,b). FISH experiments with the $18 \mathrm{~S}$ rDNA probe showed 2 clusters of rDNA genes, located interstitially on the long arm of the submetacentric neo-X and the acrocentric neo-Y chromosome (Fig. 1b). Weakly DAPI+, thick $\mathrm{CMA}_{3}+$, and double $\mathrm{C}$-positive bands were located in the paracentromeric region on the submetacentric neo-X, while thick C-positive, DAPI-, and $\mathrm{CMA}_{3}+$ blocks were found in that region on the neo-Y (Fig. 2a). Both sex chromosomes carried a secondary constriction located interstitially (Fig. 1a).

In early prophase (pachytene-diplotene), the short arm of the bi-armed neo-X, which is derived from the original $\mathrm{X}$ chromosome, showed typical positive heteropycnosis, whereas the arm which is homologous with the neo-Y presented euchromatic characteristics (Fig. 3a). C-banding and silver staining showed that in diplotene the neo-XY bivalent formed 3 to 5 chiasmata (Figs 3b, c, d). In anaphase I, the sex chromosomes always segregated to the opposite poles (Fig. 3e). Thus in metaphase II, there were 12 chromosomes - including the neo-X or the neo-Y, respectively (Fig. 3f). Two active NORs, both interstitially located, on the long arm of the neo-X and acrocentric neo-Y (Fig. 3d) were coincident with rDNA-FISH clusters (Fig. 3c).

In turn, both sex chromosomes of $S$. talpa were acrocentric; the neo-X chromosome was nearly equal in size to the largest autosome $(17.18 \%$ of TCL), whereas the neo$\mathrm{Y}$ was longer than the medium autosome pair $3(4.1 \%$ of TCL) (Fig. 1c, d). Interstitial (located closer to the distal end) thick/double C-positive, DAPI-, thick CG-rich blocks were found on the neo-Y chromosome Fig. 2b).

Only the ancestral X part of the acrocentric neo-X showed positive heteropycnosis from prophase to metaphase II 

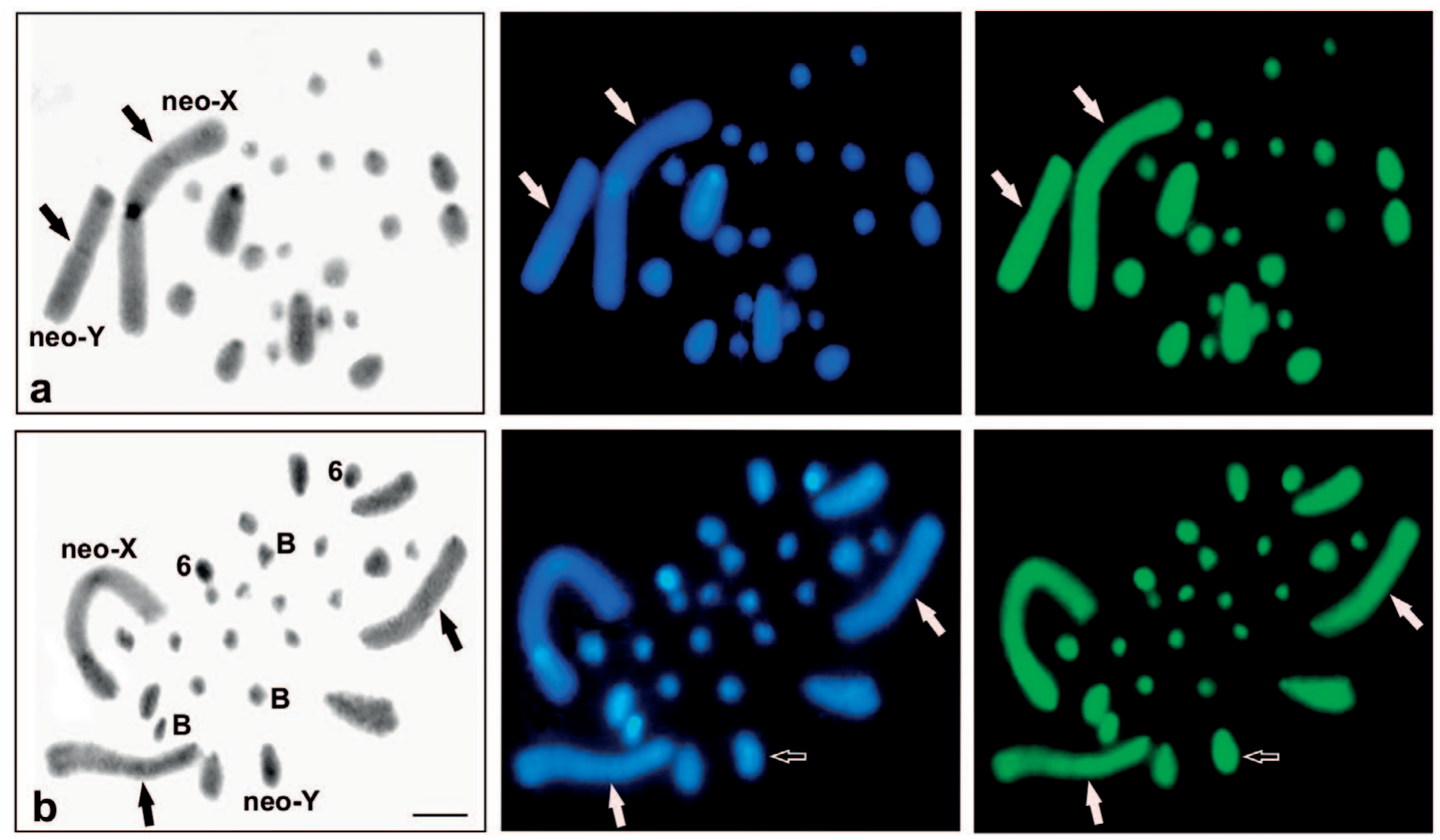

Fig. 2. C-banded and fluorochrome stained heterochromatin in spermatogonial metaphase with C-bands shown in the left panel, DAPI bands (blue) in the middle panel, and CMA 3 bands (green) in the right panel. In S.verruciferus (a) thick C-bands and weakly homogenous DAPI+/CMA + bands are visible in paracentromeric regions; in both sex chromosomes black arrows indicate a weak Cband and white arrows indicate DAPI-/CMA + bands. S. talpa (b) exhibits a very thin interstitial C-band (black arrows) and DAPI-/ weak $\mathrm{CMA}_{3}+$ bands (white arrows) on the long pair of autosomes, thick C-positive/DAPI+/CMA + bands on the chromosome pair 6 , interstitial thick/double C-band and a DAPI-/thick CG-rich block on the neo-Y chromosome (open arrows). B chromosomes (B) show very weak C-positive/DAPI $+/ \mathrm{CMA}_{3}+$ blocks. $\mathrm{Bar}=10 \mu \mathrm{m}$.

(Fig. 4a, c, d, g, h', j). In pachytene, the neo-X and neo-Y chromosomes probably exhibited partial synapsis only at their terminal parts, and a large region remained unpaired forming a loop, which suggests that the sex chromosomes are not homologous (Fig. 4b). From early diplotene to metaphase II, the sex chromosomes always (based on analysis of 80 cells) showed end-to-end association (Fig. 4c-k). This configuration occurred between both paracentromeric regions of the sex chromosomes with thin C-bands (Fig. $4 \mathrm{~g}, \mathrm{j})$. Additionally, the neo-Y always displayed an interstitially located C-band near the distal end (Fig. 4c, d, j). In anaphase I, the neo-XY bivalent always underwent an equational segregation pattern, whereas the first meiotic division was reductional for autosomes (Fig. 4h, h'). As a result, in metaphase II 12 chromosomes were observed, all cells including the heteromorphic neo-XY pair, where an association between both chromosomes in the pericentromeric regions was clearly seen (Fig. 4i-k). In anaphase II, the neo-X and neo-Y chromosomes segregated to opposite poles, giving equal numbers of X-bearing and Y-bearing spermatids (Fig. 41); no abnormal segregations were found. Consequently, the second meiotic division was reductional for the sex chromosomes and equational for the autosomes.

\section{B chromosomes}

B chromosomes (Bs) were found in most cells of all $S$. talpa specimens examined. They were similar in size to small autosomes, acrocentric (B1) or bi-armed (B2) with paracentromeric C-bands (Fig. 1c, d) and very weakly developed DAPI $+/ \mathrm{CMA}_{3}+$ blocks at their distal ends (Fig. $2 \mathrm{~b})$. They were mitotically and meiotically unstable with inter-individual variation. In spermatogonial metaphase (analyzed in all individuals) and somatic metaphase (analyzed only in two samples), the number of B1+B2 (the morphology of these chromosomes often could not be identified) varied from zero (absence of Bs) to 3. The numbers of Bs observed in spermatogonial and somatic cells in the two analyzed specimens were similar (Table 1).

The behaviour of the two types of Bs $(\mathrm{B} 1, \mathrm{~B} 2)$ during meiosis is probably connected with the appearance of the neo-XY system. Both types showed negative heteropycnosis in early meiotic prophase. In eight of the 60 analyzed cells, association between B-chrosomomes and the neo$\mathrm{XY}$ bivalent was observed from pachytene to anaphase I. This connection was observed in the part of the neo-X homologous with the neo-Y. Sometimes, the Bs were located near this bivalent (Fig. 4b-e), or independently (Fig. 4d, f, $\mathrm{h}, \mathrm{i}, \mathrm{k}$ ). Both Bs were visible as univalents or bivalents (no trivalents were observed), and in anaphase I they segregated similarly to the autosomes, but not always.

\section{DISCUSSION}

Although different tribes of the subfamily Hetrodinae have also recently been studied by us (Warchałowska- 

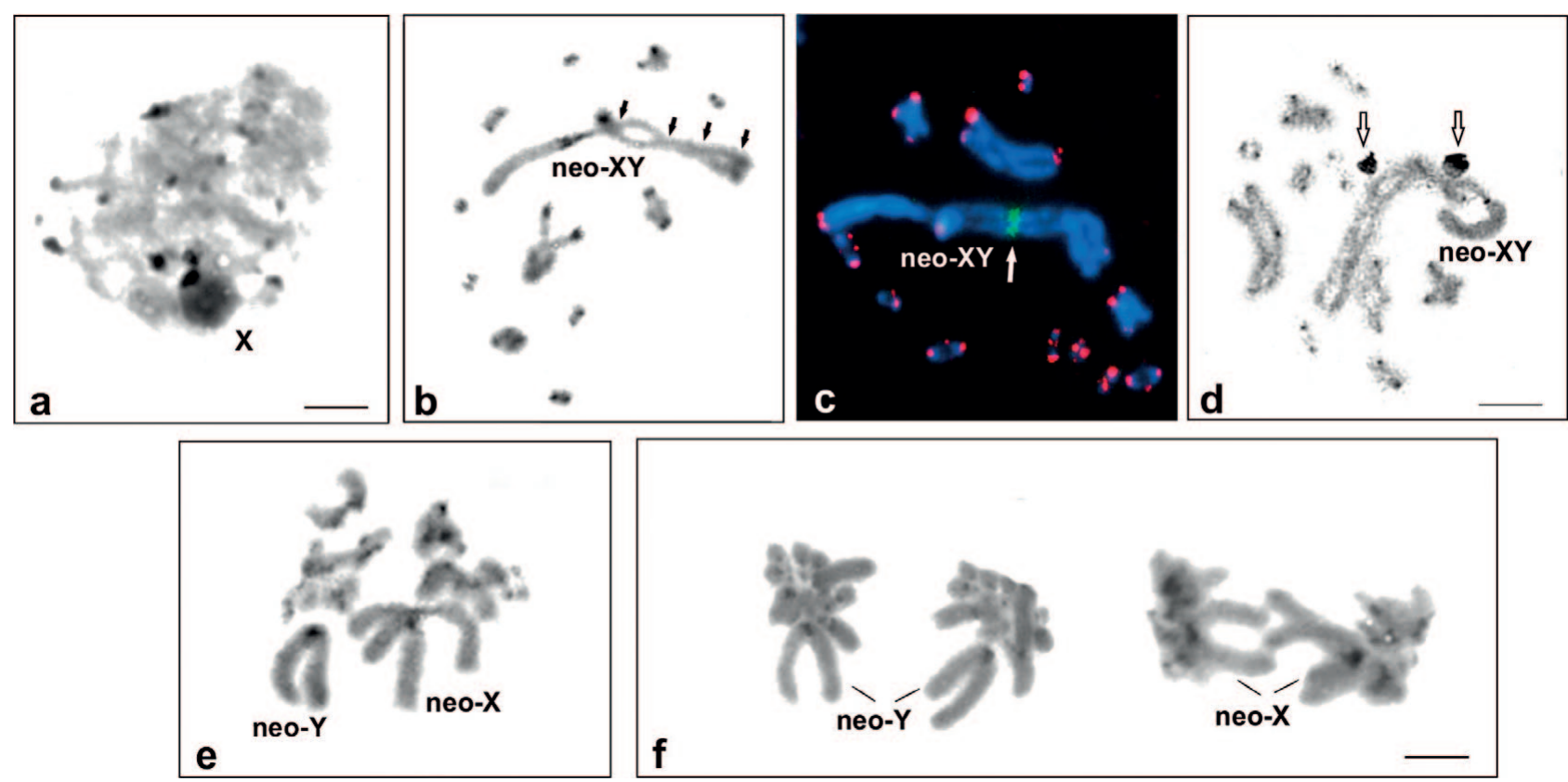

Fig. 3. The neo-XY system of S.verruciferus males subject to C-banding (a, b, e, f), silver staining (d) and FISH with both 18S rDNA (green) and telomeric DNA (red) probes (c). The original X is positively heteropycnotic in zygotene (a), and the neo-XY bivalent forms chiasmata (black arrows) in diplotene (b). rDNA signals on the neo-XY are indicated with white arrows (c) and open arrows indicate the presence of active NORs located on the neo-XY bivalent (d) in diplotene. In anaphase I (e), the sex chromosomes always segregate to opposite poles and in metaphase II (f) there are 12 chromosomes, including the neo-X or neo-Y. Bar $=10 \mu \mathrm{m}$.

Śliwa et al., in prep.), the findings concerning the two species of Spalacomimus as discussed in the present work provide important information on chromosome evolution. The diversity of diploid numbers found (from 29 to 24 in males) and the type of sex determination suggests a derived condition, similar to that in most tettigoniids. Usually males show a diploid chromosome number of $2 n=31$ acrocentric chromosomes, and an X0/XX sex chromosome system (e.g., White, 1973; Warchałowska-Śliwa, 1998). In Hetrodinae, similarly to phaneropterids in the tribe Odonturini (Warchałowska-Śliwa et al., 2011), the karyotype evolution mechanism involves a reduction in the chromosome number, including a tandem fusion (all chromosomes are acrocentric). Chromosome evolution in the two studied Spalacomimus species is interesting and appears to involve an independent origin of each of the sex chromosomes. If one assumes the ancestral chromosome number to be 31 , both species show the same processes of reduction in the chromosome number to $2 \mathrm{n}=24$. Probably multiple tandem fusions involving various autosomes and/or sex chromosomes resulted in the reduced number of acrocentric chromosomes. Analysis of the mean relative length of autosomes (see Results) shows differences between the number of long and medium chromosome pairs in both species. However, due to the lack of sufficient karyotype analysis on a larger number of specimens, it is impossible to determine which chromosomes fused. Only using chromosomal markers (like other repetitive DNAs, e.g. multigene families or single copy genes) could this question probably be answered. The origin of distinct sex chromosome systems might act as an effective barrier to isolate populations and contribute to the speciation process sympatrically (Palacios-Gimenez et al., 2013). On the other hand, it cannot be excluded that these events may have occurred before or after the divergence of both species. Therefore, Spalacomimus species represent unique models for sex chromosome differentiation research. Similar karyotype differences, including changes in chromosome number and development of three different sex chromosome systems, have been observed between closely related Phaneropterinae species of the genus Odontura (Odonturini) (Warchałowska-Śliwa et al., 2011).

TABLE 1. The occurrence of B chromosomes (B1 + B2) in spermatogonial (sp) and somatic (so) mitotic complements of $S$. talpa.

\begin{tabular}{|c|c|c|c|c|c|c|}
\hline Mitosis & Samples & Cells without B & Cells with $1 \mathrm{~B}$ & Cells with 2 B & Cells with 3 B & Total cells \\
\hline \multirow{5}{*}{$\mathrm{sp}$} & $\mathrm{CH} 7354$ & 24 & 10 & 0 & 0 & 34 \\
\hline & $\mathrm{CH} 7625$ & 0 & 10 & 0 & 0 & 10 \\
\hline & CH7626 & 19 & 17 & 1 & 6 & 43 \\
\hline & CH7699 & 0 & 0 & 12 & 9 & 21 \\
\hline & Total & 43 & 37 & 13 & 15 & 108 \\
\hline \multirow{3}{*}{ so } & CH7626 & 16 & 30 & 1 & 1 & 48 \\
\hline & $\mathrm{CH} 7699$ & 0 & 0 & 18 & 15 & 33 \\
\hline & Total & 16 & 30 & 19 & 16 & 81 \\
\hline
\end{tabular}



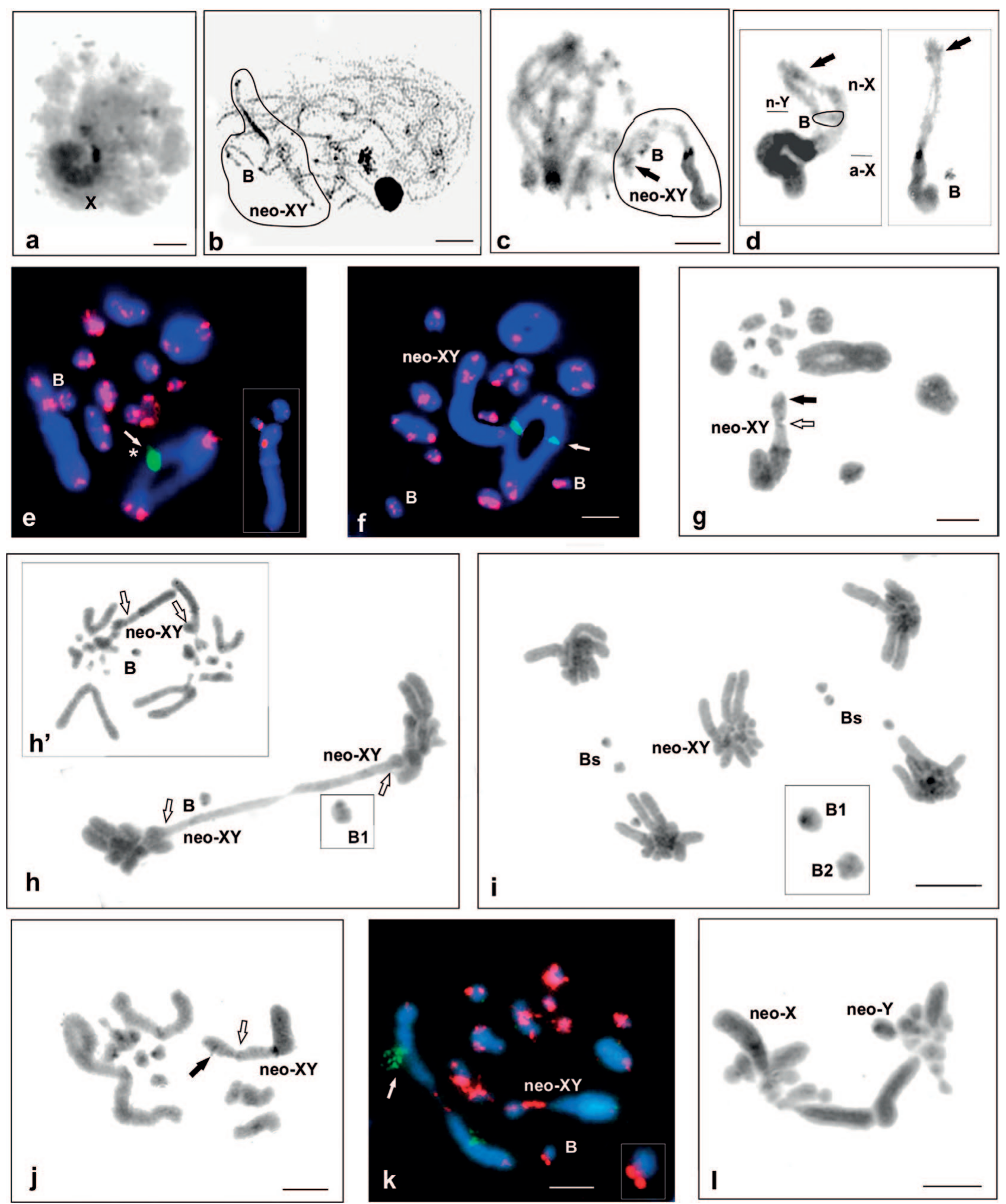

Fig. 4. The behaviour of sex chromosomes and B chromosomes (B) from pachytene to anaphase II in S. talpa males: C-banding (a, c, d, g, h, h', i, j, l), silver staining (b), and FISH with both 18S rDNA (green) and telomeric DNA (red) probes (e, f, k). The ancestral $X(a-X)$ shows positive heteropycnosis from pachytene to metaphase II (a, c, d, g, h', j), while the neo-part of X (n-X) and neo-Y (n-Y) are euchromatic ( $\mathrm{d}$ - left panel). In pachytene (b), the neo-X/neo-Y chromosomes form a loop, indicating a partial absence of homology. From diplotene to metaphase II ( $\mathrm{c}-\mathrm{k}$ and, for the selected bivalent, $\mathrm{d}$ ), there is end-to-end association of sex chromosomes between both paracentromeric regions with thin C-bands (open arrows - g, j); the neo-Y exhibits a C-band located interstitially, near the distal end (black solid arrows - c, d, g, j). White solid arrows indicate one rDNA cluster on the large bivalent $(\mathrm{e}, \mathrm{f}, \mathrm{k})$; an asterisk shows differences in the size of hybridization signals between homologous chromosomes (e). In anaphase I, the neo-XY bivalent undergoes an equational segregation pattern ( $\mathrm{h}, \mathrm{h}$ '); as a result, in metaphase II there are 12 chromosomes with a heteromorphic neo-XY pair (i-k). At anaphase II, both sex chromosomes segregate to opposite poles (l). B chromosomes are located near the sex chromosome bivalent (b, c, d - left panel, e) or independently ( $\mathrm{d}$ - right panel, $\mathrm{f}, \mathrm{h}, \mathrm{h}$, , i, k); the inset shows an enlarged image of Bs (e, $\mathrm{k})$. Bar $=10 \mu \mathrm{m}$. 


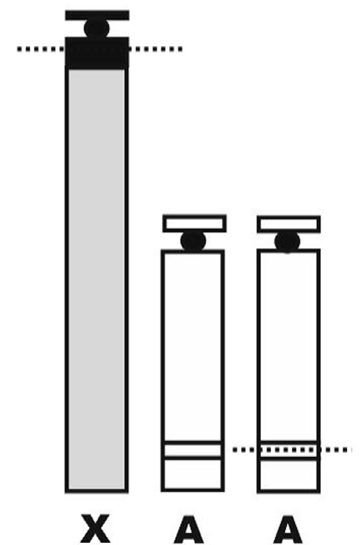

a

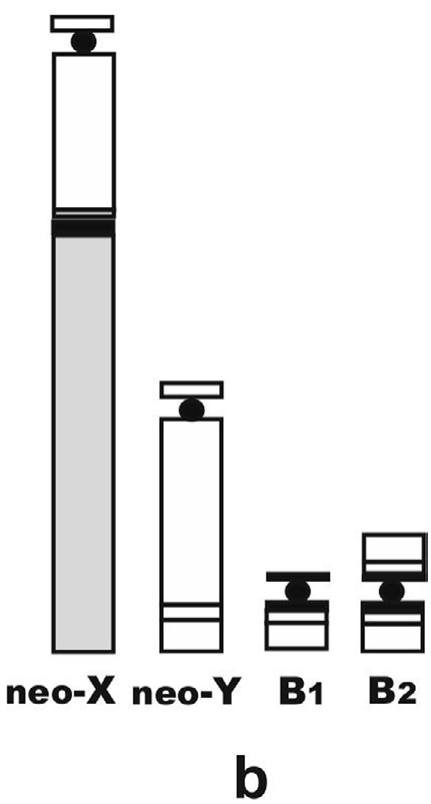

Fig. 5. A hypothetical scheme of the evolution of the neo-XY system in $S$. talpa from X0 (a) as a result of translocation between the ancestral $\mathrm{X}$ chromosome $(\mathrm{X})$ and a medium-sized autosomal pair (AA), as well as the origin of B chromosomes (B1, B2) resulting from rearrangements of the neo-sex chromosomes (b); dashed lines indicate the break point (a).

\section{Karyotypes and sex chromosome evolution}

\section{S. verruciferus}

The occurrence of thick C-bands in the paracentromeric region that fluoresced brightly upon both DAPI and $\mathrm{CMA}_{3}$ staining suggests that AT and GC bases were either situated near each other or changed positions within heterochromatin. While NOR/rDNA clusters were not detected with the fluorochrome $\mathrm{CMA}_{3}$ method, a GC-rich heterochromatin associated with this region was recognized, similar to that described in some tettigoniids, e.g., Saginae (Warchałowska-Śliwa et al., 2009), Bradyporinae (Warchałowska-Śliwa et al., 2013b), and Phaneropterinae (Grzywacz et al., 2014).

Differences in the intensity of hybridization signals produced by FISH with the (TTAGG) ${ }_{n}$ probe between the homologues of the long autosome pair in $S$. verruciferus may be related to the presence of a different number of telomeric repeats due to unequal meiotic crossing over, translocation rearrangements or homologous recombination. Similar differences in other tettigoniid have been described, for example, in some species of Barbitistini (Warchałowska-Śliwa et al., 2013a; Grzywacz et al., 2014) Bradyporinae (Warchałowska-Śliwa et al., 2013b) or Phaneropterinae (Grzywacz et al., 2011).

In $S$. verruciferus, just as in most Orthoptera, the neo-XY system is derived from a Robertsonian fusion between the ancestral acrocentric $\mathrm{X}$ (in the $\mathrm{X} 0$ system) and a long pair of acrocentric autosomes bearing an rDNA/NOR locus in the interstitial region, resulting in a bi-armed $\mathrm{X}$ and an acrocentric neo-Y chromosome. A long autosome of this type occurs in S. talpa (present study). Based on both C-banding and fluorochrome staining, the paracentromeric region of both $S$. verruciferus sex chromosomes differed in terms of heterochromatin quantity: the neo-X chromosomes showed homogenous $\mathrm{C}+/ \mathrm{DAPI}+/ \mathrm{CMA}_{3}+$ bands, whereas the neo$\mathrm{Y}$ chromosome displayed $\mathrm{C}+/ \mathrm{DAPI} / \mathrm{CMA}_{3}+$ bands. These differences are probably connected to the origin of the neo-XY. The presence of active NOR and rDNA loci only on the sex chromosome(s) has been previously observed only in some bushcricket species (Phaneropterinae), e.g., Odontura (Warchałowska-Śliwa et al., 2011), Isophya and Poecilimon (Grzywacz et al., 2014), and grasshoppers (Cabrero \& Camacho, 2008).

The neo-Y in $S$. verruciferus seems to be homologous throughout its entire length with the long arm of the neo-X. This homology is demonstrated by the same position of the rDNA-FISH signal and the GC-rich region, by complete synapsis in meiotic prophase, and by the formation of 3-5 chiasmata between the neo-Y and neo-X, which are not distally located. A similar system, with one or two chiasmata, has previously been described in Listroscelidinae (Chlorobalius leucoviridis Tepper, referred to as Yorkella picta Carl) and Phaneropterinae (Theudoria melanocnemis (Stål) by White et al. (1967a, b). Similarly as in monocentric systems, the neo-XY bivalent in $S$. verruciferus is characterized by reductional segregation during the first meiotic division and equational segregation during the second meiotic division.

\section{S. talpa}

In $S$. talpa, thin C-bands in the paracentromeric region of chromosomes (except for one small pair with a thick Cband) were CG-rich but AT-negative, similarly as in some Barbitistini bushcricket genera (Warchałowska-Śliwa et al., 2013a). 18S rDNA loci coincide with active NORs and a weak $\mathrm{CMA}_{3}+$ /DAPI- band is located interstitially on the long acrocentric bivalent. The presence of interstitial rDNA loci only on a single bivalent of bi-armed or acrocentric autosomes has previously been observed in some Bradyporinae karyotypes, as the reduction of chromosome number results from a Robertsonian translocation or tandem fusion (Warchałowska-Śliwa et al., 2013b). Consequently, in $S$. talpa the presence of a secondary constriction and the location of rDNA/NOR on the long autosome probably indicate karyotype rearrangements, which supports the hypothesis of tandem fusion.

The origin of the neo-XY in S. talpa involved more steps than that described for S. verruciferus (Fig. 5). In bushcrickets, the neo-X has been found to be produced by a tandem fusion of an autosome with the interstitial part of the original $\mathrm{X}$ in three cases: Neocallicrania selligera (Charpentier) [referred as Callicrania seoanei (Bolivar)] (Fernández-Piqueras et al., 1981), Isophya hemiptera Bey-Bienko (Warchałowska-Śliwa \& Bugrov, 1998), and Odontura arcuata Messina (Warchałowska-Śliwa et al., 2011). Possible rearrangements involved in the evolution of the neo-XY sex system from the X0 system and in the origin of B chromosomes are shown in Fig. 5 for $S$. talpa. The neo-XY system probably resulted from a fusion between the ancestral acrocentric $\mathrm{X}$ (in the $\mathrm{X} 0$ system) and a medium-sized autosome pair with thick (double) C-bands 
near the distal end. In this case, the acrocentric neo-X resulted from an interstitial break in the ancestral $\mathrm{X}$, a loss of the paracentromeric region with a C-band, and translocation of the acrocentric autosome without its distal part (with only a thin C-band). The neo-Y, a medium member of the set, is partially homologous to the autosome, and slightly larger than the homologue of the neo-X (with a double Cband). This hypothesis is based on the following observations: (1) a double C-band in the interstitial region occurs on the acrocentric neo- $\mathrm{X}$ and near the distal end of the neo$\mathrm{Y}$; (2) the size of the autosomal part translocated to the neo-X chromosome is only slightly smaller than the size of the neo-Y (3.43\% TCL and 4.1\% TCL, respectively); and (3) in meiotic stages B chromosomes (B1, B2) are often situated near the neo-XY bivalent, so it cannot be excluded that the genetic material lost during rearrangements turned into supernumerary elements. It is not possible at this stage to explain the origin of the two types of B chromosomes (B1, B2), as to do this it would be necessary to compare the DNA sequences shared by autosomal, ancestral $\mathrm{X}$ and Bs. Sex chromosomes have previously been proposed as ancestors of B chromosomes (e.g., White, 1973; Camacho et al., 2000; Cabrero et al., 2003).

In grasshoppers and bushcrickets, the neo-X chromosomes identified in species P45b, Morabinae (White et al., 1967a) as well as in Neocallicrania selligera (Charpentier) [referred to as Callicrania selligera (Bolivar)]; (Fernández-Piqueras et al., 1981) and Isophya hemiptera Bey-Bienko (Warchałowska-Śliwa \& Bugrov, 1998), both Phaneropterinae, were produced by a tandem fusion of an autosome with the interstitial part of the original $\mathrm{X}$, with the neo-X and neo-Y undergoing a post-reductional division. Here, only one chiasma occurred at an interstitial or proximal position as a consequence of a tandem fusion. Pairing between them was restricted to very short nearcentromeric heterochromatic segments of both sex chromosomes. Similar meiotic behaviour of XY chromosomes has been reported in arvicolid rodent species (MegíasNogales et al., 2003). In such rodent species, crossing over and chiasma formation between both sex chromosomes are required for correct XY segregation (Ashley et al., 1989). In $S$. talpa, during early meiotic prophase the sex chromosomes probably exhibit synaptic behaviour. However, only very short terminal parts are synapsed in the sex bivalent, while the interstitial region formed a loop due to a lack of homology, similar to that previously observed in heteropteran sex chromosomes (Bressa et al., 2009). Given the behaviour of the neo-X and neo-Y from early diplotene to metaphase I, where this bivalent shows end-to-end associations, it cannot be excluded that a chiasma could form between the distal C-band/GC-rich blocks of the sex chromosomes, but this observation is limited by cytological resolution. A post-reductional system of sex chromosomes, in which they associate achiasmatically forming a pseudobivalent, has also been described in most hymenopterans with holocentric chromosomes (e.g., Rebagliati \& Mola, 2010; Kuznetsova et al., 2011).
In the present study, sex chromosome divergence between two closely related Spalacomimus species represent a unique and useful model for the study of sex chromosome differentiation in species-specific characters. Similar karyotype differences including changes in chromosome number and development of different sex chromosome systems evolved independently ( $\mathrm{X} 0$, neo-XY, neo- $\mathrm{X}_{1} \mathrm{X}_{2} \mathrm{X}_{3}$ ), as also observed between closely related species of the phaneropterine genus Odontura (Warchałowska-Śliwa et al., 2011). So, probably in both Spalacomimus and Odontura, the turnover of sex chromosomes create markers allowing discrimination of species and phylogenetic lineages as well as for understanding the genomic diversification, speciation and evolution of the tettigoniids.

In summary, the presented cytogenetic study of two Spalacomimus species indicates that the karyotypes of this genus have undergone intensive evolution, including changes in the length of autosomes and the development of two neo-XY sex chromosome systems. While, as expected, $\mathrm{X}$-autosome Robertsonian translocations have taken place at a more or less constant rate in the evolutionary history of the neo-XY in grasshoppers and tettigoniids (including $S$. verruciferus, present study), neo-X chromosomes produced by a tandem fusion are very rare in Orthoptera. $S$. talpa is only the second known case in this group of insects in which analysis of the meiotic behaviour of the neo-X and neo-Y chromosomes has demonstrated their post-reductional division. Thus, the neo-XY sex chromosomes, which originated by different and complicated rearrangements with autosomes, their behaviour during meiosis, and the absence of heterochromatinization represent a unique model of early stages of differentiation. In the two species investigated, analysis of different populations would be necessary to explain the origin, transmission, and dynamics of supernumerary chromosomes. The present results concerning heterochromatin composition (C-banding and fluorochrome staining) and rDNA/NOR distribution, reported for the first time in Hetrodinae, prove the suitability of cytogenetic markers for distinguishing species and (probably) phylogenetic lineages. Thus, our data are a first step towards elucidating chromosome evolution in this interesting group of insects.

ACKNOWLEDGEMENTS. The authors are very grateful to K.G. Heller for fixation materials.

\section{REFERENCES}

Alicata P., Messina A. \& Oliveri S. 1974: Determinismo cromosomico del sesso in Odontura stenoxipha (Orth., Phaneropteridae): un nuovo casa di neo-XY. - Animalia 1: 109-122.

Ashley T., JaArola M. \& Fredga K. 1989: Absence of synapsis during pachynema of the normal sized sex chromosomes of Microtus arvalis. - Hereditas 111: 295-304.

Brown J.D. \& O’NeILl R.J. 2010: Chromosomes, conflict, and epigenetics: chromosomal speciation revisited. - Annu. Rev. Genomics Hum. Genet. 11: 291-316.

Bressa M.J., Papeschi A.G., VítKová M., KubíčKová S., Fuková I., Pigozzi M.I. \& Marec F. 2009: Sex chromosome evolution in cotton stainers of the genus Dysdercus (Heteroptera: Pyrrhocoridae). - Cytogenet. Genome Res. 125: 292-305. 
Bugrov A.G. \& WarchalowsKa-Śliwa E. 1997: Chromosome numbers and C-banding patterns in some Pamphagidae grasshoppers (Orthoptera, Acrididae) from the Caucasus, Central Asia, and Transbaikalia. — Folia Biol. (Kraków) 45: 133-138.

CABrero J. \& CAMACHO J.P.M. 2008: Location and expression of ribosomal RNA genes in grasshoppers: Abundance of silent and cryptic loci. - Chromosome Res. 16: 595-607.

Cabrero J., Bakkali M., Bugrov A.G., Warchalowska-Śliwa E., Lopez-Leon M.D., Perfectti F. \& Camacho J.P.M. 2003: Multiregional origin of $\mathrm{B}$ chromosomes in the grasshopper $E y$ prepocnemis plorans. - Chromosoma 112: 207-211.

CAmacho J.P.M., Orozco J.C. \& Pascual F. 1981: Chromosomal rearrangements and karyotype evolution in Decticinae (Orthoptera: Tettigonioidea). - Cytologia 46: 209-215.

Camacho J.P.M., Sharbel T.F. \& Beukeboom L.W. 2000: Bchromosome evolution. - Phil. Trans. R. Soc. Lond. (B) 355: 163-178.

Castillo E.R.D., Martí D.A. \& Bidau C.J. 2010: Sex and neosex chromosomes in Orthoptera: a review. - J. Orthopt. Res. 19: $213-231$.

Chopard L. \& KeVAn D.K. McE. 1954: Orthoptera - Ensifera from northern Kenya and Jubaland. - Tran. R. Entomol. Soc. Lond. 105: 315-353.

Dave M.J. 1965: On unusual sex chromosomes found in two species of the Locustidae. - Cytologia 30: 194-200.

FARIA R. \& NAVARRO A. 2010: Chromosomal speciation revisited: rearranging theory with pieces of evidence. - Trends Ecol. Evol. 25: 660-669.

FAVRELLE M. 1936: Contribution à l'étude de la garniture chromosomique des Orthoptères (Gryllidae et Tettigoniidae). - Mém. Mus. R. Hist. Nat. Belg. (Ser. 2) 3: 53-60.

FERreira A. 1969: Chromosome survey of some Australian tettigoniids (Orthoptera, Tettigonioidea): Two species with neo-XY sex determining mechanism. - Cytologia 34: 511-522.

Ferreira A. 1976: Cytology of Brazilian Phaneropteridae (Orthoptera, Tettigonnioidea): a species with neo XY sex determining mechanism. - Can. J. Genet. Cytol. 18: 79-84.

Fernández-Piqueras J., Rojo García E. \& Sentís Castaño C. 1981: A tandem fusion origin of a neo XY sex determining mechanism in the long-horned Callicrania seoanei (Bol.). Heredity 48: 147-150.

Fernández-Piqueras J., Rodríguez Camos A., Sentís Castaño C. \& Wandosell JuRAdo F. 1982: Pycnogaster cucullata (Charp.): a polytypic species of Tettigonioidea with $\mathrm{X} 0$ and neo XY sex determination. - Heredity 48: 147-150.

Grzywacz B., Chobanov D.P., Maryańska-Nadachowska A., Karamysheva T.V., Heller K.-G. \& Warchalowska-Śliwa E. 2014: A comparative study of genome organization and inferences for the systematics of two large bushcricket genera of the tribe Barbitistini (Orthoptera: Tettigoniidae: Phaneropterinae). - BMC Evol. Biol. 14: 48.

Hemp C. 2013: Annotated list of Ensifera (Orthoptera) and further records on Caelifera (Orthoptera) of Mt Kilimanjaro, Tanzania. - Zootaxa 3613: 301-342.

Kitano J. \& Peichel C.L. 2012: Turnover of sex chromosome and speciation in fishes. - Environ. Biol. Fishes 94: 549-558.

Král J., Musilová J., ŠŤáhlavskÝ F., Řezáč M., Akan Z., Edwards R.L., Coyle F.A. \& AlmerJe C.B. 2006: Evolution of the karyotype and sex chromosome systems in basal clades of araneomorph spider (Aranea: Araneomorphae). - Chromosome Res. 14: 859-880.

Kuznetsova V.G., Grozeva S.M., Nokkala S. \& Nokkala C. 2011: Cytogenetics of the true bug infraorder Cimicomorpha (Hemiptera, Heteroptera): a review. - ZooKeys 154: 31-70.
Matthey R. 1948: Données nouvelles sur les chromosomes des Tettigonides et la parthenogenèse de Saga pedo Pallas. — Rev. Suisse Biol. 55: 45-56.

МватA K.J. 2005: A contribution to the cytogenetics of the armoured ground cricket, Acanthoplus speiseri (Orthoptera: Tettigoniidae), a pest of grain crops in Zambia. - Int. J. Trop. Insect Sci. 25: 142-145.

Messina A. 1981: Sulle specie di Odontura del gruppo Stenoxypha (Fieb.) (Orthoptera, Phaneropterinae). - Animalia 8: 15-23.

Megías-Nogales B., Marchal J.A., Acosta M.J., Bullejos M., Díaz de la Guardia R. \& SÁnchez A. 2003: Sex chromosomes pairing in two Arvicolidae species: Microtys nivalis and Arvicola sapidus. - Hereditas 138: 114-121.

Nguyen P., SÝkorová M., Šíchová J., Kưta V., Dalíková M., Čapková Frydrychová R., Neven L.G., Sahara K. \& Marec F. 2013: Neo-sex chromosomes and adaptive potential in tortricid pests. - Proc. Natl. Acad. Sci. USA 110: 6931-6936.

Palacios-Gimenez O.M., Castillo E.R., Marti D.A. \& CabralDE-Mello D.C. 2013: Tracking the evolution of sex chromosome system in Melanoplinae grasshoppers through chromosomal mapping of repetitive DNA sequences. - BMC Evol. Biol. 13: 167.

Rebagliati P.J. \& Mola L.M. 2010: Meiotic behaviour and karyotypic variation in Acledra (Pantatomidae, Heteroptera). — Genet. Mol. Res. 9: 739-749.

SchweIzer D. 1976: Reverse fluorescent chromosome banding with chromomycin and DAPI. - Chromosoma 58: 307-324.

SuMNER S.G. 1972: A simple technique for demonstrating centromere heterochromatin. - Exp. Cell. Res. 75: 304-306.

Ueshima N. \& Rentz D.C. 1979: Chromosome systems in the North American Decticinae with references to robertsonian changes (Orthoptera: Tettigoniidae). - Cytologia 44: 693714 .

WarchalowSKA-ŚliwA E. 1998: Karyotype characteristics of katydid orthopterans (Ensifera, Tettigoniidae) and remarks on their evolution at different taxonomic levels. - Folia Biol. (Kraków) 46: 143-176.

WarchalowsKa-Śliwa E. \& Bugrov A.G. 1998: Karyotypes and C-banding patterns of some Phaneropterinae katydids (Orthoptera, Tettigonioidea) with special attention to a post-reductional division of the neo- $\mathrm{X}$ and the neo- $\mathrm{Y}$ sex chromosomes in Isophya hemiptera. — Folia Biol. (Kraków) 46: 47-54.

WarchalowsKa-Śliwa E. \& Bugrov A.G. 2009: Karyotype of the South African katydid Hetrodes pupus (Linnaeus, 1758) (Orthoptera, Tettigoniidae) with special reference to relationships within the Hetrodinae subfamily. - Zootaxa 2137: 43-50.

WARChA£OWSKA-ŚLIWA E. \& MARYAŃSKA-NADACHOWSKA A. 1992: Karyotypes, C-bands, NORs location in spermatogenesis of Isophya brevipennis Brunner (Orthoptera: Phaneropteridae). - Caryologia 45: 83-89.

WarchalowsKa-Śliwa E., GrZywacz B., MaryańsKa-Nadachowska A., Karamysheva T.V., Rubtsov N.B. \& Chobanov D.P. 2009: Chromosomal differentiation among bisexual European species of Saga Charp. (Orthoptera, Tettigoniidae, Saginae) detected by both classical and molecular methods. - Eur. J. Entomol. 106: 1-9.

Warchalowska-Śliwa E., MaryańsKa-Nadachowska A., Grzywacz B., Karamysheva T., Lehmann A.W., Lehmann G.U.C. \& Heller K.-G. 2011: Changes in the numbers of chromosomes and sex determination system in bushcrickets of the genus Odontura (Orthoptera, Tettigoniidae, Phaneropterinae). - Eur. J. Entomol. 108: 183-195.

WarchalowsKa-Śliwa E., Grzywacz B., Maryańska-Nadachowska A., Karamysheva T., Heller K.-G., Lehmann A.W., Leh- 
MANN G.U.C. \& ChoBAnov D.P. 2013a: Molecular and classical chromosomal techniques reveal diversity in bushcricket genera of Barbitistini (Orthoptera). - Genome 56: 667-676.

Warchałowska-Śliwa E., Grzywacz B., Maryańska-Nadachowska A., Karamysheva T., Chobanov D.P. \& Heller K.-G. 2013b: Cytogenetic variability among Bradyporinae species (Orthoptera: Tettigoniidae). - Eur. J. Entomol. 110: 1-12.

Webber B.J., Rentz D.C., Ueshima N. \& Woodrow I.E. 2003: Leucopodoptera eumundii, a new genus and species of katydid from the tropical rainforest of North Queensland, Australia (Orthoptera: Tettigoniidae: Phaneropterinae: Holochlorini). J. Orthopt. Res. 12: 79-88.

Weidner H. 1955: Die Hetrodinae (Orthoptera, Saltatoria). Mitt. Hambg. Zool. Mus. Inst. 53: 109-172.
White M.J.D. 1973: Animal Cytology and Evolution. 3rd ed. Cambridge University Press, London, 961 pp.

White M.J.D. 1979: Karyotypes and meiosis of the morabine grasshoppers. II. The genera Culmacris and Stiletta. - Aust. J. Zool. 27: 109-133.

White M.J.D., Blackith R.E., Blackith R.M. \& Cheney J. 1967a: Cytogenetics of the viatica group of morabinae grasshoppers. I. The "coastal" species. - Aust. J. Zool. 15: 263-302.

White M.J.D., Mesa A. \& Mesa R. 1967b: Neo-XY sex chromosome mechanisms in two species of Tettigonioidea (Orthoptera). - Cytologia 32: 190-199.

Received July 2, 2014; revised and accepted October 23, 2014 Prepublished online December 5, 2014 